\title{
Interatrial Shunt Device for Heart Failure With Preserved Ejection Fraction
}

\begin{abstract}
David M. Kaye* and Shane Nanayakkara
Heart Failure Research Group, Department of Cardiology, Baker Heart and Diabetes Institute, Alfred Hospital, Melbourne, VIC, Australia
\end{abstract}

Heart failure with preserved ejection fraction (HFpEF) accounts for approximately half of the current burden of HF, and the prevalence is continuing to rise. In contrast to HF with reduced ejection fraction (HFrEF) there are no clinically effective evidence based therapies for HFpEF. The principal pathophysiologic disorder is an elevation of left atrial pressure, most notable during physical activity, which results from impaired left ventricular diastolic reserve, and increased left atrial stiffness. This review outlines the clinical development of a potential device based therapy for HFpEF, the interatrial shunt device (IASD).

Keywords: heart failure, diastolic dysfunction, structural heart device, clinical trial, cardiac catheter interventional treatment

Heart failure (HF) is one of the most prevalent cardiovascular disorders and recent data highlight a continuing rise in the number of people living with HF, most notably in the context of an aging population (1). In particular, incident HF with preserved ejection fraction (HFpEF), defined by a left ventricular ejection fraction of $>50 \%$, has emerged over recent years to be at least equal in importance to that of $\mathrm{HF}$ with reduced ejection fraction (HFrEF) (2). In older persons, particularly in women, HFpEF is rapidly emerging as the most prevalent form of HF (3). This epidemiologic transition, reflects the rising prevalence both non-modifiable and modifiable risk factors such as aging, hypertension, diabetes, and obesity $(2,4-6)$. The increased diagnosis also reflects increased clinical focus on HFpEF and the availability of diagnostic tools, such as echocardiography and natriuretic peptide assays. The functional limitation and reduction in quality of life experienced by HFpEF patients is similar to that in patients with $\operatorname{HFrEF}(7,8)$. Overall survival in HFpEF patients is worse than that in a healthy age-matched population (9-12) and in several studies similar to that of HFrEF. Currently, HFpEF treatment represents one of the most challenging problems in cardiovascular medicine, given that to date, pharmacological treatments with proven effectiveness in HFrEF, have failed to demonstrate benefit in large scale clinical trials (13-22).

As outlined in Figure 1, the pathophysiologic basis of HFpEF is complex and the clinical features represent the integrated effect of a range of abnormalities in cardiac, vascular and non-cardiovascular comorbidities. Elevated left atrial (LA) pressure is considered to be the hallmark feature of HFpEF, often only becoming apparent during physical activity (23-25). We previously showed that although the resting pulmonary capillary wedge pressure (PCWP) tends to be greater in HFpEF patients than in healthy control subjects and patients classified with non-cardiac dyspnea (NCD) $(26,27)$, the PCWP is often within the proscribed "normal" range. However, in contrast to normal subjects or NCD patients, individuals with HFpEF display a characteristic marked rise in PCWP at low workload (Figure 2). The magnitude of the rise in PCWP during physical activity has been demonstrated to correlate with functional capacity and with outcomes $(25,28)$. 


Cardiac
Left Ventricle
: Stiffness, relaxation
- Contractility
Left atrium
- Stiffness, relaxation
- Contractility
Atrial Fibrillation
Chronotropic Reserve
Right Ventricle
- Contractility
Myocardial Flow Reserve
Dynamic MR

Vascular

Systemic Circulation

- Stiffness

- VACoupling

- Oxygen Delivery

Pulmonary Circulation

- Stiffness

- VACoupling
Co-Morbidities

- Diabesity

- Aging

- COPD

- CKD

- Frailty

FIGURE 1 | Schematic represents the pathophysiology of heart failure with preserved ejection fraction.

Various mechanisms contribute to the disproportionate, exercise mediated rise in LA pressure in HFpEF including increased left ventricular and LA stiffness together with impaired active relaxation $(27,29,30)$. The cause of abnormal ventricular diastolic and atrial mechanics is complicated. Although left ventricular hypertrophy has been incorporated into diagnostic algorithms for HFpEF (6), its present is not at all uniform in HFpEF (31). Myocardial fibrosis is commonly observed in HFpEF (32) and likely contributes substantially to increased ventricular and atrial stiffness. However, the capacity pharmacological interventions to substantially reverse fibrosis appears to be limited, at least in the short to intermediate term (33). Aside from fibrosis as therapeutic target in HFpEF, pharmacologic interventions directed toward pathways that influence diastolic performance by improved myocardial blood flow, active relaxation, or cardiomyocyte stiffness have also been studied. In particular, a substantial range of clinical studies of agents directed toward the nitric oxide/cyclic guanosine monophosphate/protein kinase $\mathrm{G}$ pathway have been conducted $(17,18,21,22,34)$, however none have yielded clear benefit.

In the absence of effective drug therapies for HFpEF, a devicebased approach to reduce LA pressure, especially in the context of the exertional rise, has been developed. This principle was based upon the observation that patients the combination of mitral stenosis and a congenital atrial septal defect (Lutembacher's syndrome) are less symptomatic than patients with isolated mitral stenosis of similar severity due to LA pressure offloading. Based upon invasive hemodynamic data obtained from patients with symptomatic $\mathrm{HF}$ and an $\mathrm{LVEF} \geq 40 \%$, computer modeling studies were conducted to determine the optimal size of an interatrial shunt that would result in a lower peak LA pressure, whilst avoiding excessive left to right shunting (35). As shown in Figure 3, simulation studies identified that an $8 \mathrm{~mm}$ interatrial shunt would substantially attenuate the activity based rise in LA pressure, whilst creating only a small resting left to right shunt of 1.3-1.4:1.

Based on these computations an interatrial shunt device (IASD, Corvia Medical, Tewksbury, MA, USA) was developed to be positioned within the atrial septum and to provide continuous left to right shunting through an $8 \mathrm{~mm}$ central lumen (Figure 4). The implant procedure requires trans-septal catheterization and the passage of a 16Fr delivery sheath. The trans-septal puncture is performed using standard techniques, supported by either transesophageal or intra-cardiac echocardiography. The delivery catheter is advanced over a guide wire into the LA, followed by deployment of the left side of the IASD (Figure 4C). Subsequently, the delivery system is retracted to cause the opened arms of the IASD to abut the inter-atrial septum on the left atrial side of the septum. Following confirmation of apposition to the septum, the right side of the device is deployed, thereby locating the IASD in position. IASD patients are treated with lifelong aspirin together with short-term (typically 3-6 months) clopidogrel. Experience with other similar devices such as the "V-Wave" system has also been reported (36).

Following an initial pilot study of the IASD which confirmed safety together with evidence of improved hemodynamics (37) a multi-center, open-label, non-randomized study of the IASD was conducted with clinical, echocardiographic, and hemodynamic follow-up after 6 months and 1 year $(38,39)$. Patients with known HFpEF were included if they had evidence 
of chronic symptomatic HF (NYHA class II-IV), a left ventricular ejection fraction $>40 \%$ and an elevated PCWP at rest $(>15$ $\mathrm{mmHg}$ ) or during exercise $(>25 \mathrm{mmHg}$ ) measured by right heart catheterization. Patients with significant right ventricular dysfunction including a central venous pressure $(\mathrm{CVP})>14$ $\mathrm{mmHg}$ and tricuspid annular plane systolic excursion (TAPSE) $<14 \mathrm{~mm}$ were excluded to reduce the potential risk of right heart

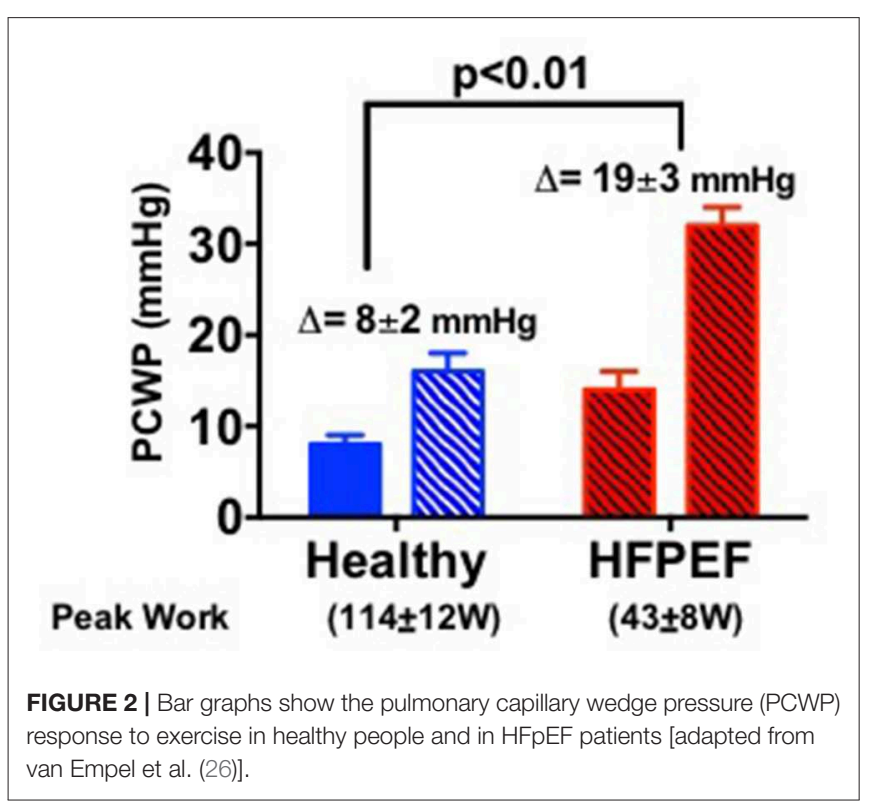

failure and to ensure that left atrial pressure exceeded right atrial pressure to ensure continued left-to-right interatrial shunting. The IASD was successfully implanted in 64 patients and there were no device related complications. At 6 months, in this open label study, there was evidence of significant improvement in quality of life measures and functional status and evidence of an improvement in the peak exercise PCWP especially when corrected for workload (38). Oximetric assessment of the left to right shunt revealed a Qp:Qs of $\sim 1 \mathrm{~g}: 1$, consistent with the prior modeling work. IASD implantation was associated with a modest but significant increase in right atrial pressure, right atrial volume, and right ventricular volume together with an increase in right sided cardiac output (38). At the 1 year follow up there was evidence of sustained clinical benefit with regards to quality of life, functional capacity and the hemodynamic profile whilst the echocardiographic features of right sided remodeling were stable (39). Evidence of hemodynamic benefit, with a reduction in PCWP with the IASD during exercise has recently been confirmed in a small randomized, parallel group, blinded study (40).

These encouraging data have prompted the commencement of a pivotal multi-center randomized clinical trial (REDUCE LAP HF-II, NCT03088033) of 608 patients, with hemodynamically confirmed HFpEF, randomized to IASD implant, or sham procedure and followed for 5 years. Cross over will be allowed at 24 months. The primary endpoint of the study is a composite of the incidence of cardiovascular mortality or ischemic stroke, the rate of heart failure admissions or healthcare facility visits for IV diuresis, and a change in the KCCQ

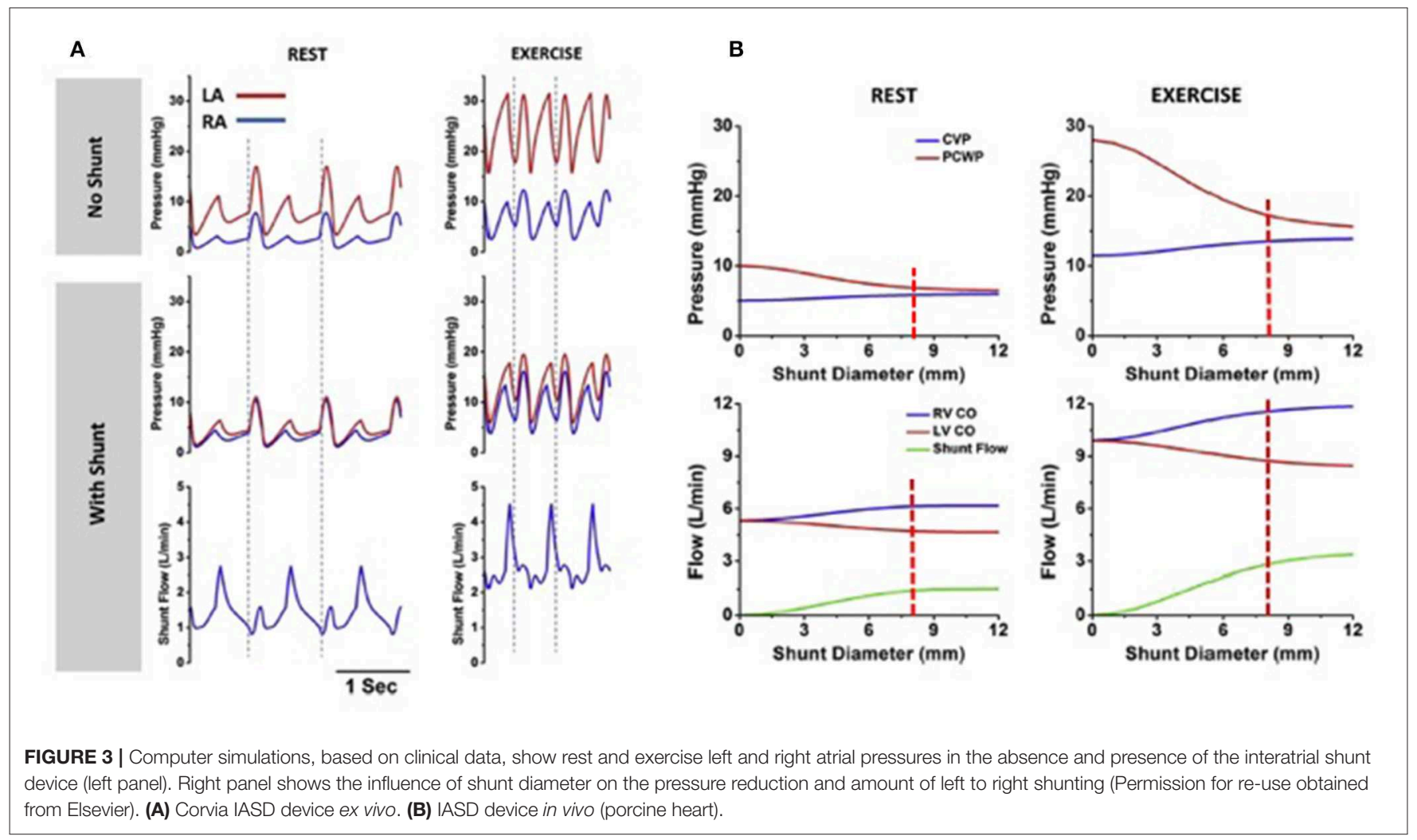



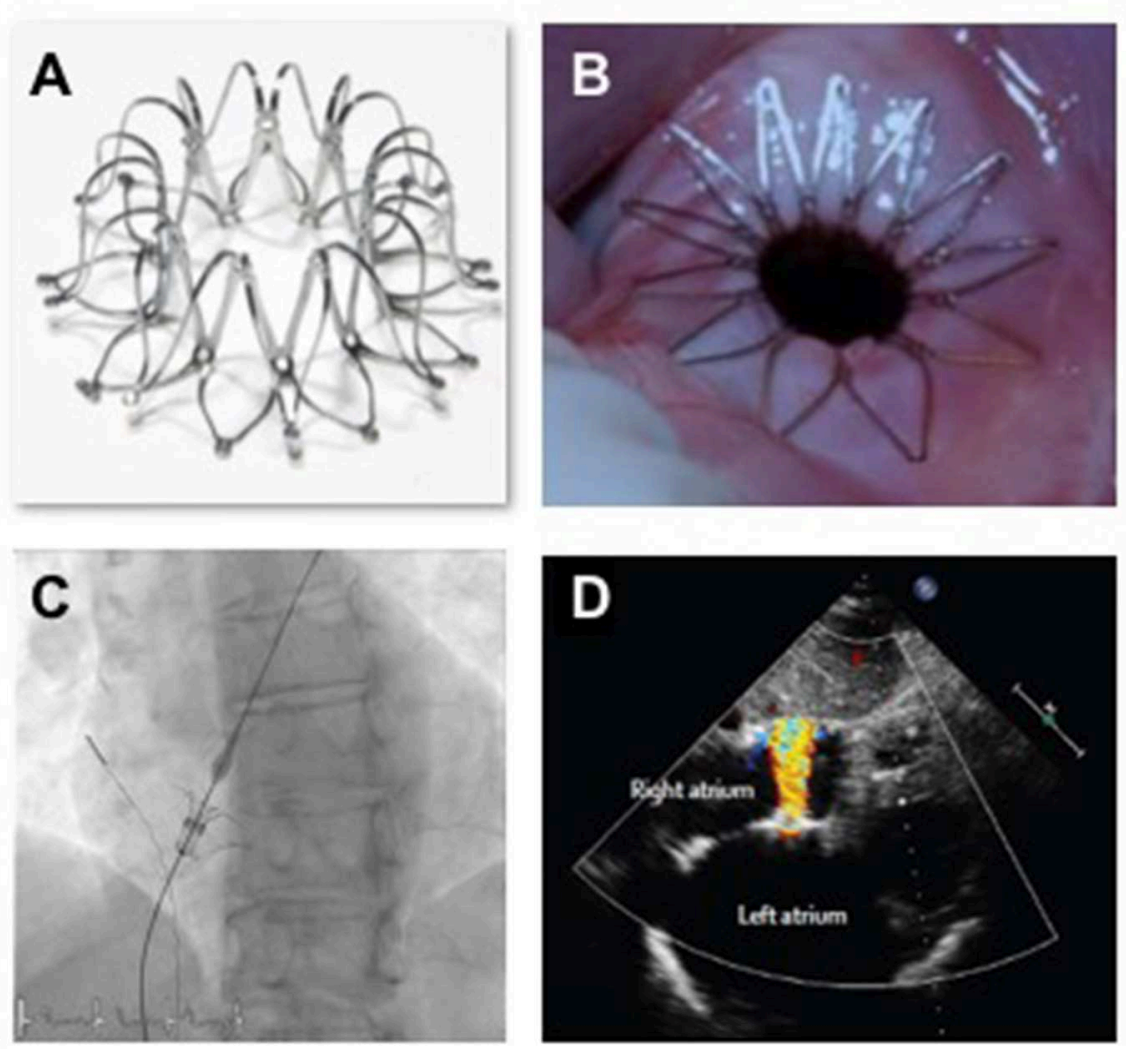

FIGURE 4 | Images (A,B) show the IASD; (C) show the fluoroscopic image of IASD delivery; (D) echocardiographic image showing left to right blood flow.

total summary score. In this context, it is relevant to consider the nature of overall and cardiovascular mortality in patients with HFpEF. Epidemiologic studies highlight the complex, multimorbid nature of HFpEF patients in which mortality relates predominantly to cardiovascular events, however with a significant contribution from non-cardiovascular events (12).

An alternate device, the V-Wave System (V-Wave, Caesarea, Israel) has also been trialed in HF. The device is an hourglass shaped self-expanding prosthesis on a nitinol frame, with a polytetrafluoroethylene (PTFE) skirt extending across both sides of the septum. The central lumen is smaller than the Corvia device $(5.1 \mathrm{~mm})$, and includes a porcine trileaflet valve on the right atrial side designed to prevent right-to-left flow. All patients were anticoagulated for 3 months post implantation. Thirty-eight patients were enrolled in a single arm feasibility study, with $100 \%$ initial implant success. All forms of heart failure were included, with $8(21 \%)$ having an ejection fraction over $40 \%$. Half of the devices were either occluded or stenotic by 12 months, with some evidence of improved hemodynamics in those with patent devices. Further randomized data in the HFrEF and HFpEF population are required following further device modification to improve shunt patency.

The precise nature and type of cardiovascular mortality in HFpEF is not well-characterized, given the limitations of available datasets, however it appears to result from a combination of heart failure, MI, sudden death, and stroke. The potential effect of IASD implantation on HF outcomes has been investigated recently by modeling the predicted outcome of the REDUCE LAP-HF cohort in the absence of the IASD using the MAGGIC prognostic model $(41,42)$, and comparing it to actual outcomes over a 3 years follow-up period (43). Although this study was small in size and there were relatively few death, it was shown that the observed mortality was significantly lower than that predicted. Further post-hoc analysis in this study also demonstrated that patients who had a particularly favorable hemodynamic response to IASD implantation had lower rates of HF hospitalization. The potential for IASD implantation to impact on survival is consistent with the study by Dorfs et al. who showed that a higher workload correct exercise PCWP was associated with poorer survival (28).

Given the complex nature of HFpEF and the invasive nature of IASD implantation, as compared to pharmacotherapy, it is appropriate to consider those patients who might benefit most from the procedure. Given that the IASD implant functions to reduce LA pressure, attempts have been made to better understand the factors that contribute most to LA pressure particularly during exercise. Wessler et al. demonstrated that the magnitude of the reduction in exercise PCWP is influenced by the baseline pressure gradient between left and right atria (44). As such, the pressure of right heart failure and elevated 
right atrial pressure is of particular concern in patients being considered for IASD implantation. In conjunction, it may also possible to identify patients that may derive the greatest benefit by predict those that would have the greatest elevation of exercise LA pressure. Telles et al. have recently shown that patients with more marked reductions in LA strain appear to have the greatest increase in exercise PCWP and therefore might derive greater benefit (27). An increased rate of atrial fibrillation is also a potential consequence of a device inserted in the atrial septum; conversely, a reduction in left atrial pressure may also reduce the risk of $\mathrm{AF}$.

Taken together, the management of HFpEF patients continues to be a major, unresolved clinical challenge. Despite the complexity of the pathophysiological pathways leading to the development of HFpEF, the fundamental final consequence is an elevation in left atrial pressure. The IASD was developed

\section{REFERENCES}

1. Conrad N, Judge A, Tran J, Mohseni H, Hedgecott D, Crespillo AP, et al. Temporal trends and patterns in heart failure incidence: a population-based study of 4 million individuals. Lancet. (2018) 391:572-80. doi: 10.1016/S0140-6736(17)32520-5

2. Dunlay SM, Roger VL, Redfield MM. Epidemiology of heart failure with preserved ejection fraction. Nat Rev Cardiol. (2017) 14:591-602. doi: 10.1038/nrcardio. 2017.65

3. Beale AL, Meyer P, Marwick TH, Lam CSP, Kaye DM. Sex differences in cardiovascular pathophysiology: why women are overrepresented in heart failure with preserved ejection fraction. Circulation. (2018) 138:198-205. doi: 10.1161/CIRCULATIONAHA.118.034271

4. Owan TE, Hodge DO, Herges RM, Jacobsen SJ, Roger VL, Redfield MM. Trends in prevalence and outcome of heart failure with preserved ejection fraction. N. Engl. J. Med. (2006) 355:251-9. doi: 10.1056/NEJMoa052256

5. Bhatia RS, Tu JV, Lee DS, Austin PC, Fang J, Haouzi A, et al. Outcome of heart failure with preserved ejection fraction in a population-based study. $\mathrm{N} \mathrm{Engl} \mathrm{J}$ Med. (2006) 355:260-9. doi: 10.1056/NEJMoa051530

6. Ponikowski P, Voors AA, Anker SD, Bueno H, Cleland JG, Coats AJ, et al. 2016 ESC Guidelines for the diagnosis and treatment of acute and chronic heart failure: the task force for the diagnosis and treatment of acute and chronic heart failure of the European Society of Cardiology (ESC). Developed with the special contribution of the Heart Failure Association (HFA) of the ESC. Eur J Heart Fail. (2016) 18:891-975. doi: 10.1002/ejhf.592

7. Kitzman DW. Exercise intolerance. Prog Cardiovasc Dis. (2005) 47:367-79. doi: 10.1016/j.pcad.2005.02.002

8. Lewis EF, Lamas GA, O’Meara E, Granger CB, Dunlap ME, McKelvie RS, et al. Characterization of health-related quality of life in heart failure patients with preserved versus low ejection fraction in CHARM. Eur J Heart Fail. (2007) 9:83-91. doi: 10.1016/j.ejheart.2006.10.012

9. Tribouilloy C, Rusinaru D, Mahjoub H, Souliere V, Levy F, Peltier M, et al. Prognosis of heart failure with preserved ejection fraction: a 5 years prospective population-based study. Eur Heart J. (2008) 29:339-47. doi: 10.1093/eurheartj/ehm554

10. Lee DS, Gona P, Vasan RS, Larson MG, Benjamin EJ, Wang TJ, et al. Relation of disease pathogenesis and risk factors to heart failure with preserved or reduced ejection fraction: insights from the framingham heart study of the national heart, lung, and blood institute. Circulation. (2009) 119:3070-7. doi: 10.1161/CIRCULATIONAHA.108.815944

11. Chan MMY, Lam CSP. How do patients with heart failure with preserved ejection fraction die? Eur. J. Heart Failure. (2013) 15:604-13. doi: 10.1093/eurjhf/hft062

12. Vaduganathan M, Patel RB, Michel A, Shah SJ, Senni M, Gheorghiade M, et al. Mode of death in heart failure with preserved ejection fraction. J Am Coll Cardiol. (2017) 69:556-69. doi: 10.1016/j.jacc.2016.10.078 to specifically address this physiologic target and early studies have shown the device to be safe and with promising evidence of efficacy. A pivotal randomized controlled study, REDUCE LAP-HF II is currently underway to definitely determine the clinical utility of this procedure.

\section{AUTHOR CONTRIBUTIONS}

All authors listed have made a substantial, direct and intellectual contribution to the work, and approved it for publication.

\section{FUNDING}

SN was supported by a Heart Foundation scholarship, grant number 101116.
13. Yusuf S, Pfeffer MA, Swedberg K, Granger CB, Held P, McMurray JJ, et al. Effects of candesartan in patients with chronic heart failure and preserved left-ventricular ejection fraction: the CHARM-Preserved Trial. Lancet. (2003) 362:777-81. doi: 10.1016/S0140-6736(03)14285-7

14. Cleland JGF, Tendera M, Adamus J, Freemantle N, Polonski L, Taylor J. The perindopril in elderly people with chronic heart failure (PEP-CHF) study. Eur. Heart J. (2006) 27:2338-45. doi: 10.1093/eurheartj/ehl250

15. Pitt B, Pfeffer MA, Assmann SF, Boineau R, Anand IS, Claggett B, et al. Spironolactone for heart failure with preserved ejection fraction. N Engl J Med. (2014) 370:1383-92. doi: 10.1056/NEJMoa1313731

16. van Veldhuisen DJ, Cohen-Solal A, Bohm M, Anker SD, Babalis D, Roughton $\mathrm{M}$, et al. Beta-blockade with nebivolol in elderly heart failure patients with impaired and preserved left ventricular ejection fraction: data from SENIORS (Study of effects of nebivolol intervention on outcomes and rehospitalization in seniors with heart failure). J Am Coll Cardiol. (2009) 53:2150-8. doi: 10.1016/j.jacc.2009.02.046

17. Redfield MM, Anstrom KJ, Levine JA, Koepp GA, Borlaug BA, Chen HH, et al. Isosorbide mononitrate in heart failure with preserved ejection fraction. $N$ Engl J Med. (2015) 373:2314-24. doi: 10.1056/NEJMoa1510774

18. Redfield MM, Chen HH, Borlaug BA, Semigran MJ, Lee KL, Lewis G, et al. Effect of phosphodiesterase-5 inhibition on exercise capacity and clinical status in heart failure with preserved ejection fraction: a randomized clinical trial. JAMA. (2013) 309:1268-77. doi: 10.1001/jama.2013.2024

19. Borlaug BA, Koepp KE, Melenovsky V. Sodium nitrite improves exercise hemodynamics and ventricular performance in heart failure with preserved ejection fraction. J Am Coll Cardiol. (2015) 66:1672-82. doi: 10.1016/j.jacc.2015.07.067

20. Zamani P, Rawat D, Shiva-Kumar P, Geraci S, Bhuva R, Konda P, et al. Effect of inorganic nitrate on exercise capacity in heart failure with preserved ejection fraction. Circulation. (2015) 131:371-80. doi: 10.1161/CIRCULATIONAHA.114.012957

21. Nanayakkara S, Kaye D. Targets for heart failure with preserved ejection fraction: therapeutic targets for HFPEF. Clin Pharmacol Ther. (2017) 102:22837. doi: $10.1002 / \mathrm{cpt} .723$

22. Borlaug BA, Anstrom KJ, Lewis GD, Shah SJ, Levine JA, Koepp GA, et al. Effect of inorganic nitrite vs placebo on exercise capacity among patients with heart failure with preserved ejection fraction: the INDIE-HFpEF randomized clinical trial. JAMA. 320, 1764-73. doi: 10.1001/jama.2018.14852

23. Maeder MT, Thompson BR, Brunner-La Rocca HP, Kaye DM. Hemodynamic basis of exercise limitation in patients with heart failure and normal ejection fraction. J Am Coll Cardiol. (2010) 56:855-63. doi: 10.1016/j.jacc.2010. 04.040

24. Borlaug BA, Nishimura RA, Sorajja P, Lam CSP, Redfield MM. Exercise hemodynamics enhance diagnosis of early heart failure with preserved ejection fraction. Circulation. (2010) 3:588-95. doi: 10.1161/CIRCHEARTFAILURE.109.930701 
25. Wolsk E, Kaye D, Borlaug BA, Burkhoff D, Kitzman DW, Komtebedde J, et al. Resting and exercise haemodynamics in relation to six-minute walk test in patients with heart failure and preserved ejection fraction. Eur J Heart Fail. (2018) 20:715-22. doi: 10.1002/ejhf.976

26. van Empel VP, Mariani J, Borlaug BA, Kaye DM. Impaired myocardial oxygen availability contributes to abnormal exercise hemodynamics in heart failure with preserved ejection fraction. J Am Heart Assoc. (2014) 3:e001293. doi: 10.1161/JAHA.114.001293

27. Telles F, Nanayakkara S, Evans S, Patel HC, Mariani JA, Vizi D, et al. Impaired left atrial strain predicts abnormal exercise haemodynamics in heart failure with preserved ejection fraction. Eur J Heart Fail. (2019) 21:495-505. doi: 10.1002/ejhf.1399

28. Dorfs S, Zeh W, Hochholzer W, Jander N, Kienzle RP, Pieske B, et al. Pulmonary capillary wedge pressure during exercise and long-term mortality in patients with suspected heart failure with preserved ejection fraction. Eur Heart J. (2014) 35:3103-12. doi: 10.1093/eurheartj/ehu315

29. Westermann D, Kasner M, Steendijk P, Spillmann F, Riad A, Weitmann K, et al. Role of left ventricular stiffness in heart failure with normal ejection fraction. Circulation. (2008) 117:2051-60. doi: 10.1161/CIRCULATIONAHA.107.716886

30. Melenovsky V, Hwang SJ, Redfield MM, Zakeri R, Lin G, Borlaug BA. Left atrial remodeling and function in advanced heart failure with preserved or reduced ejection fraction. Circ Heart Fail. (2015) 8:295-303. doi: 10.1161/CIRCHEARTFAILURE.114.001667

31. Solomon SD, Zile M, Pieske B, Voors A, Shah A, Kraigher-Krainer E, et al. The angiotensin receptor neprilysin inhibitor LCZ696 in heart failure with preserved ejection fraction: a phase 2 double-blind randomised controlled trial. Lancet. (2012) 380:1387-95. doi: 10.1016/S0140-6736(12)61227-6

32. Mohammed SF, Hussain S, Mirzoyev SA, Edwards WD, Maleszewski JJ, Redfield MM. Coronary microvascular rarefaction and myocardial fibrosis in heart failure with preserved ejection fraction. Circulation. (2015) 131:550-9. doi: 10.1161/CIRCULATIONAHA.114.009625

33. Kitzman DW, Hundley WG, Brubaker PH, Morgan TM, Moore JB, Stewart KP, et al. A randomized double-blind trial of enalapril in older patients with heart failure and preserved ejection fraction: effects on exercise tolerance and arterial distensibility. Circulation. (2010) 3:477-85. doi: 10.1161/CIRCHEARTFAILURE.109. 898916

34. Pieske B, Maggioni AP, Lam CSP, Pieske-Kraigher E, Filippatos G, Butler $\mathrm{J}$, et al. Vericiguat in patients with worsening chronic heart failure and preserved ejection fraction: results of the SOluble guanylate Cyclase stimulatoR in heArT failurE patientS with PRESERVED EF (SOCRATESPRESERVED) study. Eur Heart J. (2017) 38:1119-27. doi: 10.1093/eurheartj/ ehw593

35. Kaye D, Shah SJ, Borlaug BA, Gustafsson F, Komtebedde J, Kubo S, et al. Effects of an interatrial shunt on rest and exercise hemodynamics: results of a computer simulation in heart failure. J Card Fail. (2014) 20:212-21. doi: 10.1016/j.cardfail.2014. 01.005

36. Del Trigo M, Bergeron S, Bernier M, Amat-Santos IJ, Puri R, CampeloParada F, et al. Unidirectional left-to-right interatrial shunting for treatment of patients with heart failure with reduced ejection fraction: a safety and proof-of-principle cohort study. Lancet. (2016) 387:1290-7. doi: 10.1016/S0140-6736(16)00585-7

37. Sondergaard L, Reddy V, Kaye D, Malek F, Walton A, Mates M, et al. Transcatheter treatment of heart failure with preserved or mildly reduced ejection fraction using a novel interatrial implant to lower left atrial pressure. Eur J Heart Fail. (2014) 16:796-801. doi: 10.1002/ejhf.111

38. Hasenfuss G, Hayward C, Burkhoff D, Silvestry FE, McKenzie S, Gustafsson $\mathrm{F}$, et al. A transcatheter intracardiac shunt device for heart failure with preserved ejection fraction (REDUCE LAP-HF): a multicentre, open-label, single-arm, phase 1 trial. Lancet. (2016) 387:1298-304. doi: 10.1016/S0140-6736(16)00704-2

39. Kaye DM, Hasenfuss G, Neuzil P, Post MC, Doughty R, Trochu JN, et al. One-year outcomes after transcatheter insertion of an interatrial shunt device for the management of heart failure with preserved ejection fraction. Circ Heart Fail. (2016) 9:e003662. doi: 10.1161/CIRCHEARTFAILURE.116. 003662

40. Feldman T, Mauri L, Kahwash R, Litwin S, Ricciardi MJ, van der Harst $P$, et al. Transcatheter interatrial shunt device for the treatment of heart failure with preserved ejection fraction (REDUCE LAP-HF I [Reduce elevated left atrial pressure in patients with heart failure]): a phase 2, randomized, sham-controlled trial. Circulation. (2018) 137:364-75. doi: 10.1161/CIRCULATIONAHA.117. 032094

41. Meta-analysis Global Group in Chronic Heart F. The survival of patients with heart failure with preserved or reduced left ventricular ejection fraction: an individual patient data meta-analysis. Eur Heart J. (2012) 33:1750-7. doi: 10.1093/eurheartj/ehr254

42. Pocock SJ, Ariti CA, McMurray JJ, Maggioni A, Kober L, Squire IB, et al. Predicting survival in heart failure: a risk score based on 39372 patients from 30 studies. Eur Heart J. (2013) 34:1404-13. doi: 10.1093/eurheartj/ehs337

43. Kaye DM, Petrie MC, McKenzie S, Hasenfubeta G, Malek F, Post M, et al. Impact of an interatrial shunt device on survival and heart failure hospitalization in patients with preserved ejection fraction. ESC Heart Fail. (2018) 6:62-69. doi: 10.1002/ehf2.12350

44. Wessler J, Kaye D, Gustafsson F, Petrie MC, Hasenfubeta G, Lam CSP, et al. Impact of baseline hemodynamics on the effects of a transcatheter interatrial shunt device in heart failure with preserved ejection fraction. Circ Heart Fail. (2018) 11:e004540. doi: 10.1161/CIRCHEARTFAILURE.117. 004540

Conflict of Interest Statement: The authors declare that the research was conducted in the absence of any commercial or financial relationships that could be construed as a potential conflict of interest.

Copyright (C) 2019 Kaye and Nanayakkara. This is an open-access article distributed under the terms of the Creative Commons Attribution License (CC BY). The use, distribution or reproduction in other forums is permitted, provided the original author(s) and the copyright owner(s) are credited and that the original publication in this journal is cited, in accordance with accepted academic practice. No use, distribution or reproduction is permitted which does not comply with these terms. 